\title{
SOX2 as a novel contributor of oxidative metabolism in melanoma cells
}

\author{
Elena Andreucci ${ }^{1 \dagger}$, Silvia Pietrobono ${ }^{2 \dagger}$, Silvia Peppicelli ${ }^{1}$, Jessica Ruzzolini ${ }^{1}$, Francesca Bianchini $^{1}$, Alessio Biagioni ${ }^{1}$, \\ Barbara Stecca ${ }^{2}$ and Lido Calorini ${ }^{1,3^{*}}$ (D)
}

\begin{abstract}
Background: Deregulated metabolism is a hallmark of cancer and recent evidence underlines that targeting tumor energetics may improve therapy response and patient outcome. Despite the general attitude of cancer cells to exploit the glycolytic pathway even in the presence of oxygen (aerobic glycolysis or "Warburg effect"), tumor metabolism is extremely plastic, and such ability to switch from glycolysis to oxidative phosphorylation (OxPhos) allows cancer cells to survive under hostile microenvironments. Recently, OxPhos has been related with malignant progression, chemo-resistance and metastasis. OxPhos is induced under extracellular acidosis, a well-known characteristic of most solid tumors, included melanoma.
\end{abstract}

Methods: To evaluate whether SOX2 modulation is correlated with metabolic changes under standard or acidic conditions, SOX2 was silenced and overexpressed in several melanoma cell lines. To demonstrate that SOX2 directly represses HIF1A expression we used chromatin immunoprecipitation (ChIP) and luciferase assay.

Results: In A375-M6 melanoma cells, extracellular acidosis increases SOX2 expression, that sustains the oxidative cancer metabolism exploited under acidic conditions. By studying non-acidic SSM2C and 501-Mel melanoma cells (high- and very low-SOX2 expressing cells, respectively), we confirmed the metabolic role of SOX2, attributing SOX2-driven OxPhos reprogramming to HIF1 a pathway disruption.

Conclusions: SOX2 contributes to the acquisition of an aggressive oxidative tumor phenotype, endowed with enhanced drug resistance and metastatic ability.

Keywords: Melanoma, Tumor extracellular acidosis, SOX2, HIF1a, Oxidative metabolism

\section{Background}

In the last decades, tumor metabolism has drawn increasing attention in the scientific world and deregulating cellular energetics has recently become a hallmark of cancer [1]. Instead of using an oxidative metabolism like most of normal cells, cancer cells convert glucose into lactate even in the presence of high oxygen tension, exploiting the so-called aerobic glycolysis or "Warburg effect". Despite the energetic gain in terms of ATP production is lower than during the

\footnotetext{
* Correspondence: lido.calorini@unifi.it

${ }^{\dagger}$ Elena Andreucci and Silvia Pietrobono contributed equally to this work. 'Department of Clinical and Experimental Biomedical Sciences "Mario Serio", Section of Experimental Pathology and Oncology, University of Florence, Viale G.B. Morgagni, 50, 50134 Florence, Italy

${ }^{3}$ Center of Excellence for Research, Transfer and High Education DenoTHE University of Florence, Florence, Italy

Full list of author information is available at the end of the article
}

oxidative phosphorylation (OxPhos), the Warburg metabolism is about 100-fold faster than OxPhos and ensures biomass formation and DNA duplication, that is crucial for cancer cell proliferation [2]. Indeed, fermentation to lactic acid and the glycolytic breakdown of glucose generate a number of substrates which turn into "anabolic" precursors for the synthesis of different compounds, such as glucose-6-phosphate for glycogen and ribose 5-phosphate, dihydroxyacetone phosphate for triacylglyceride and phospholipids, and pyruvate for alanine and malate. Metabolite accumulation upstream pyruvate production is further increased by the up-regulation of the low activity M2 isoform of pyruvate kinase (PKM2), that slows down the last step of glycolysis. In this respect, intermediate components of the glycolytic pathway appear to be more significant than its final product pyruvate. Given the limited 
pyruvate supply, to replenish the tricarboxylic acid cycle (TCA) cancer cells increase glutamine consumption, a key nutrient that provides carbon for acetyl-CoA, citrate production and lipogenesis, nitrogen for purine, pyrimidine and DNA synthesis, and reducing power in the form of NADPH to support cell proliferation [3]. The particular attitude of proliferating cancer cells to use aerobic glycolysis favors a microenvironment enriched in lactate and protons, with a subsequent $\mathrm{pH}$ reduction. Moreover, the large amount of lactate released by tumor cells can be taken up by normal stromal cells to regenerate pyruvate, which in turn can be extruded to refuel cancer cells [4]. The reduction in oxygen tension that characterizes proliferating tumor tissues, stimulates the hypoxia-inducible factor $\alpha$ (HIF1 $\alpha)$, which drives the anaerobic glycolysis. This leads to lactate dehydrogenase A (LDH-A)-dependent lactic acid production, and the upregulation of monocarboxylated transporter (MCT)4 and of sodium-proton exporters to avoid intracellular acidosis. As a direct consequence, both aerobic and anaerobic glycolysis adopted by cancer cells contribute to the acidification of tumor microenvironment. Dysregulated $\mathrm{pH}$ is emerging as a hallmark of cancer, since cancer cells show a 'reversed' $\mathrm{pH}$ gradient with a constitutively increased intracellular $\mathrm{pH}(\mathrm{pHi})$ that is higher than the extracellular $\mathrm{pH}(\mathrm{pHe})$. Indeed, while normal differentiated adult cells show $\mathrm{pHi}$ of $\sim 7.2$ and $\mathrm{pHe}$ of $\sim 7.4$, cancer cells have a higher $\mathrm{pHi}(>7.4)$ and a lower pHe (6.7-7.1). This 'reversed' $\mathrm{pH}$ gradient creates a perfect storm for metastatic progression [5] by promoting malignant phenotype endowed with apoptosis resistance, radio- and chemotherapy resistance, immune surveillance escape programs, increased migration and ability of secondary organs colonization [6]. As an additional aspect, we have recently reported that acidic cancer cells undergo a metabolic change characterized by the acquisition of a more OxPhos phenotype through the inhibition of HIF1 $\alpha$ expression, associated with a reduced proliferation compared to standard $\mathrm{pH}$ condition [7].

Tumor cells are extremely plastic even in terms of cellular energetics and may shift their metabolic phenotypes to adapt to microenvironmental changes, giving a selective advantage to cancer cells under unfavourable environments [8]. Most of solid tumors, including melanoma, undergo such plastic changes in metabolism. Cutaneous melanoma, despite representing less than $5 \%$ of all skin cancers, is responsible for the majority of skin cancer-related deaths [9]. The incidence of malignant melanoma in most developed countries has risen faster than any other cancer type since the mid-1950s. It is estimated that the annual increase in the incidence rate of melanoma has been approximately $3-7 \%$ per year worldwide for Caucasians. Detection and surgical treatment of early-stage disease seems to prevent progression in most cases. However, patients with deep primary tumors or tumors that metastasize to regional lymph nodes frequently develop distant metastases. Median survival after the onset of distant metastases is only 6-9 months, and the 5-year survival rate is less than 5\% [10].

Recent studies have pointed out the crucial role of the transcription factor SOX2 (sex-determining region $\mathrm{Y}$ (SRY)-Box2) in melanoma and cancer in general. SOX2 has been correlated with growth, tumorigenicity, drug resistance, and metastasis in at least 25 different tumors, including cancers of the ovary, lung, skin, brain, breast, prostate, and pancreas [11]. In the majority of these cancers, SOX2 has been found to have increased expression or gene amplification in tumor tissues. Moreover, SOX2 has been associated with stemness and tumor initiating cells (TICs), proposed to explain origin and heterogeneity of many tumors [12], including cervical, lung, ovarian, head and neck squamous cell carcinoma, medulloblastoma, skin squamous-cell carcinoma, and melanoma [11]. Indeed, SOX2 has been reported to regulate self-renewal and tumorigenicity of human melanoma-initiating cells $[13,14]$. Previous reports indicate that SOX2 is expressed in $50 \%$ of melanomas and a minority of nevi [15-17], and is associated with dermal invasion and primary tumor thickness [18]. However, the role of SOX2 in melanoma growth and progression is more controversial. While an early paper reported that SOX2 silencing reduces in vivo growth of A2058 melanoma cells [15], recent studies suggest that SOX2 is dispensable for melanomagenesis and metastasis formation $[19,20]$.

Here we show for the first time that SOX2 is highly expressed in melanoma cells exposed to extracellular acidosis, where it modulates cell metabolism in order to favor an oxidative phenotype, possibly interfering with HIF $1 \alpha$ expression. This additional attitude of SOX2 might add new information on its crucial importance in malignant progression.

\section{Methods}

\section{Cell cultures}

A375-M6 [21], commercial 501-Mel, SK-Mel-2, SKMel-5, SK-Mel-28 and patient-derived SSM2c [13] melanoma cell lines were maintained in DMEM $4.5 \mathrm{~g} / \mathrm{l}$ glucose, $2 \mathrm{mM} \mathrm{L-glutamine,} \mathrm{and} \mathrm{10 \%} \mathrm{FBS} \mathrm{(Euroclone,}$ Milan Italy). 24-h medium acidification was obtained by adding $\mathrm{HCl} 1 \mathrm{~N}$ in complete culture medium to reach $\mathrm{pH} 6.7 \pm 0.1$. $\mathrm{pH}$ value was monitored by using Orion $\mathrm{pH}$ meter 520A-1. $\mathrm{pH}$ was monitored for the first hour after medium acidification to check the 
maintenance of a $\mathrm{pH}$ value at 6.7 , and then at the end point of each experiment. Cells were treated with 50 mM 2-deoxyglucose (Calbiochem, San Diego, CA, USA) or $10 \mathrm{mM}$ Metformin (Sigma-Aldrich, Milan, Italy) for $24 \mathrm{~h}$.

\section{SOX2 silencing and overexpression}

SOX2-silenced A375-M6 cells were obtained by siRNA transfection with Sox-2 siRNA (sc-38408, Santa Cruz Biotechnology, Dallas, Texas, USA) or control siRNA-A (sc-37007, Santa Cruz Biotechnology), according to manufacturer's instructions. SOX2 silencing in SSM2c cells was obtained by lentiviral transduction. Lentiviruses were produced in HEK-293 T cells. Lentiviral vectors used were pLKO.1-puro (LV-c) (Open Biosystems, Lafayette, CO, USA) and pLKO.1-puro-shSOX2-1 (LV-shSOX2-1) targeting the 3' untranslated region of SOX2 (targeting sequence 5'-CTGCCGAGAATCCATGTATAT-3') as previously reported [13]. SOX2 overexpression in 501-Mel cells was obtained by retroviral transduction. Retroviruses were produced in HEK-293 T cells. Retroviral vectors used were generated by co-transfection of $1 \mu \mathrm{g}$ pBABE (Addgene, Cambridge, MA, USA, \#1764) or pBABE-SOX2 (cloned into the BamHI/SalI restriction sites of $\mathrm{pBABE}$ vector using the following primers: SOX2-F 5'-ATGTACAAC ATGATGGAGACGG-3' and SOX2-R 5'-TCACATGTG TGAGAGGGGC-3'), $0.9 \mu \mathrm{g}$ pUMVC packaging plasmid (Addgene, \#8449) and $0.1 \mu \mathrm{g}$ pCMV-VSV-G envelope (Addgene, \#8454).

\section{Western blot analysis}

Cells were lysed in RIPA buffer (Merck Millipore) containing PMSF (Sigma-Aldrich), sodium orthovanadate (Sigma-Aldrich), and protease inhibitor cocktail (Calbiochem), sonicated and centrifuged $15 \mathrm{~min}$ at $14,000 \mathrm{rpm}$ at $4{ }^{\circ} \mathrm{C}$. Equal amounts of protein were separated on Bolt Bis-Tris Plus gels, $4-12 \%$ precast polyacrylamide gels (Life Technologies, Milan, Italy). Fractionated proteins were transferred to a PVDF membrane using the iBlot 2 System (Life Technologies). Following 1-h blocking with Odyssey blocking buffer (Dasit Science, Milan, Italy), membrane was probed overnight at $4{ }^{\circ} \mathrm{C}$ with the following primary antibodies: anti-SOX2 mouse monoclonal antibody (R\&D System, Minneapolis, MN, USA), anti-HIF- $1 \alpha$ rabbit polyclonal antibody (Novusbio, Milan, Italy), anti- GLUT-1, GLUT-3, MCT-1, MCT-4 and PGC1 $\alpha$ rabbit polyclonal antibodies (Santa Cruz Biotechnology). After that, membrane was incubated $1 \mathrm{~h}$ at room temperature with goat anti-mouse IgG Alexa Fluor 680 antibody (Invitrogen) or goat anti-rabbit IgG Alexa Flour 750 antibody (InvitrogenLife Technologies, Milan, Italy). Membrane was visualized by the Odyssey Infrared Imaging System (LI-COR ${ }^{\circ}$ Bioscience, Lincoln, Nebraska USA). Anti-HSP90 (Santa Cruz Biotechnology), $\beta$-actin (Sigma-Aldrich) and HDAC2
(Santa Cruz Biotechnology) antibodies were used to assess equal amount of protein loaded in each lane.

\section{Flow cytometry}

Cells were harvested by using Accutase (Euroclone), collected in flow cytometer tubes $\left(2 \times 10^{5}\right.$ cells/tube), permeabilized for $15 \mathrm{~min}$ with $0.25 \%$ Tryton X-100 PBS, and incubated $1 \mathrm{~h}$ at $4{ }^{\circ} \mathrm{C}$ with anti-SOX2 antibody (Santa Cruz Biotechnology). Cells were washed in PBS and incubated $1 \mathrm{~h}$ in the dark at $4{ }^{\circ} \mathrm{C}$ with anti-goat antibody conjugated with FITC (Merk Millipore, Milan, Italy). Samples were washed in PBS and the analyzed at BD FACSCanto (BD Biosciences, Milan, Italy). The flow cytometer was calibrated using cells incubated with secondary antibody only. For each sample, $1 \times 10^{4}$ events were analysed.

\section{Lactate production}

Lactate production by cancer cells was evaluated in 24-h conditioned medium by using D-Lactate Colorimetric Assay Kit (Biovision, CA, USA) according to manufacturer's instructions. The analysis was performed at the microplate reader (Bio-Rad, Milan, Italy) and data normalized for the cell number of each sample, to get a final result of lactate production $(\mathrm{nM})$ by $1 \times 10^{5}$ cells.

\section{Glucose uptake detection}

Glucose uptake by melanoma cells was evaluated by using Glucose Uptake Cell-Based Assay Kit (Cayman Chemical, Michigan, USA) according to manufacturer's instructions. Briefly, melanoma cells were glucose-starved for $1 \mathrm{~h}$ by using RPMI medium without glucose (Euroclone), then incubated for $15 \mathrm{~min}$ in the dark with 2-NBDG, a FITC-labeled deoxyglucose analog, harvested and analyzed at BD FACSCanto (BD Biosciences). The flow cytometer was calibrated using untreated cells. For each sample, $1 \times 10^{4}$ events were analyzed.

\section{Quantitative real time PCR (qPCR)}

Total RNA was prepared using Tri Reagent (Sigma-Aldrich), agarose gel checked for integrity, and reverse transcribed with iScript cDNA Synthesis Kit (Bio-Rad) according to the manufacturer's instructions. Selected genes were evaluated by a real-time RT-PCR with 7500 Fast Real-Time PCR System (Applied Biosystems, Monza, Italy). Fold change was determined by the comparative $\mathrm{Ct}$ method using $\beta$-actin, TATA sequence binding protein (TBP), glyceraldehyde 3-phosphate dehydrogenase (GAPDH) and $\beta 2$-microglobulin as housekeeping genes. Amplification was performed with the PCR setting: 40 cycles of $95^{\circ} \mathrm{C}$ for $15 \mathrm{~s}$ and of $60^{\circ} \mathrm{C}$ for $60 \mathrm{~s}$ using PowerUp SYBR Green Master Mix (Thermo Fisher Scientific). Primer sequences (IDT, Tema Ricerca, Bologna, Italy) are listed in Table 1. 
Table 1 List of forward and reverse primers used for qPCR analysis

\begin{tabular}{|c|c|c|}
\hline Gene & Forward & Reverse \\
\hline GLUT1 & CGGGCCAAGAGTGTGCTAA & TGACGATACCGGAGCCAATG \\
\hline GLUT3 & CGAACTTCCTAGTCGGATTG & AGGAGGCACGACTTAGACAT \\
\hline HK2 & CAAAGTGACAGTGGGTGTGG & GCCAGGTCCTTCACTGTCTC \\
\hline LDHA & AGGGAATGTACGGCATTGAG & CCTCATCGTCCTTCAGCTTC \\
\hline PDK1 & CCAAGACCTCGTGTTGAGACC & $\begin{array}{l}\text { AATACACGTCTCAGGTCTC } \\
\text { CTTGG }\end{array}$ \\
\hline PDP2 & TAGGCCAACCTTTGTTTCACCA & AGACCCTCACAACAAAAGCCT \\
\hline MCT-1 & GTGGCTCAGCTCCGTATTGT & GAGCCGACCTAAAAGTGGTG \\
\hline MCT-4 & CAGTTCGAGGTGCTCATGG & ATGTAGAGGTGGGTCGCATC \\
\hline PGC1a & GGGAAAGTGAGCGATTAGTTGAG & CATGTAGAATTGGCAGGTGGAA \\
\hline CytC & TTGCACTTACACCGGTACTTAAGC & ACGTCCCCACTCTCTAAGTCCAA \\
\hline COX41 & GGCCCGGCATTTTACGA & TCACCGTGGAGCGGAAA \\
\hline $\operatorname{cox} 5 b$ & TGCGCTCCATGGCATCA & CCCAGTCGCCTGCTCTTC \\
\hline ATP5A1 & TGCAAGGACTTCCATGCCTC & CGCCCAGGTTCTTCAAGATCAA \\
\hline SOX2 2 & GAGCTTTGCAGGAAGTTTGC & GCAAGAAGCCTCTCCTTGAA \\
\hline $\begin{array}{l}\text { HIF1a } \\
\text { promoter }\end{array}$ & TGCAAAGTTGCCAAAGGCCA & CAGGGGAACTCACCTTGTCTAC \\
\hline HIF1a & GGCGCGAACGACAAGAAAAA & TCCAAATCACCAGCATCCAGA \\
\hline TBP & CAACAGCCTGCCACCTTAC & CTGAATAGGCTGTGGGGTC \\
\hline ACTIN & TCGAGCCATAAAAGGCAACT & CTTCCTCAATCTCGCTCTCG \\
\hline GAPDH & GACGCTGGGGCTGGCATTG & GCTGGTGGTCCAGGGGTC \\
\hline $\begin{array}{l}\text { B2- } \\
\text { microglobulin }\end{array}$ & GCCGTGTGAACCATGTGACT & GCTTACATGTCTCGATCCCACTT \\
\hline
\end{tabular}

\section{Annexin V/PI flow cytometer analysis}

Cell death was determined by flow cytometer analysis using Annexin V FITC-conjugated (Immunotools GmbH, Friesoythe, Germany) and PI (Sigma-Aldrich) according to the manufacturer's protocol. Briefly, cells were harvested with Accutase (Eurolone), collected in flow cytometer tubes $\left(1 \times 10^{5}\right.$ cells/tube), washed in PBS, and incubated $15 \mathrm{~min}$ at $4{ }^{\circ} \mathrm{C}$ in the dark with $100 \mu \mathrm{l}$ Annexin binding buffer (100 mM HEPES, $140 \mathrm{mM} \mathrm{NaCl}, 25 \mathrm{mM}$ $\mathrm{CaCl} 2, \mathrm{pH} 7.4), 1 \mu \mathrm{l}$ of $100 \mu \mathrm{g} / \mathrm{ml}$ PI working solution, and $5 \mu \mathrm{l}$ Annexin V FITC-conjugated. Each sample was added with Annexin binding buffer to reach $500 \mu \mathrm{l}$ volume/tube. Samples were analyzed at BD FACSCanto (BD Biosciences). Cellular distribution depending on Annexin V and/or PI positivity allowed the measure of the percentage of viable cells (Annexin V and PI negative cells), early apoptosis (Annexin V-positive and PI negative cells), late apoptosis (Annexin V and PI-positive cells), and necrosis (Annexin V-negative and PI-positive cells).

\section{Chromatin immunoprecipitation}

Melanoma cells were fixed with $1 \%$ formaldehyde for $10 \mathrm{~min}$ and lysed in cell Lysis Buffer (5 mM PIPES pH $8,85 \mathrm{mM} \mathrm{KCl}, 0.5 \% \mathrm{NP}-40$ ) added with protease inhibitors. Nuclei were collected by centrifugation at 4500 rpm for $10 \mathrm{~min}$ and lysed in nuclear lysis buffer (1\%
SDS, $10 \mathrm{mM}$ EDTA, $50 \mathrm{mM}$ Tris- $\mathrm{HCl} \mathrm{pH}$ 8) added with protease inhibitors. Chromatin was sonicated to an average size of 200-600 bp, diluted with ChIP Dilution Buffer (10 mM Tris-HCl pH 8, 1\% Triton X-100, 2 mM EDTA, $140 \mathrm{mM} \mathrm{NaCl}$ ) and incubated overnight with $20 \mu \mathrm{l}$ of protein $\mathrm{G}$ magnetic dynabeads pre-conjugated with mouse anti-SOX2 (MAB2018; R\&D System) or normal mouse IgG (sc-2025; Santa Cruz Biotechnology) antibodies. DNA was purified and qPCR was carried out at $60^{\circ} \mathrm{C}$ using FastStart SYBR Green Master (Roche Diagnostic, Monza, Italy) in a Rotorgene-Q (Qiagen, Milan, Italy). Primer sequences are listed in Table 1.

\section{Luciferase reporter assays}

Luciferase reporters were used in combination with Renilla luciferase pRL-TK reporter vector (Promega, Madison, WI) to normalize luciferase activities. pCS2 + MT vector (Promega) was used to equal DNA amounts; pCS2 + SOX2 was cloned into pCS2 + MT using the following primers: Fwd 5'-ATGTACAACATGATGGAGA CGG-3', Rev. 5'-CACATGTGTGAGAGGGGC-3' after digestion with $\mathrm{XhoI} / \mathrm{SnaBI}$ restriction enzymes; pGL4.20HIF1 $\propto$ prom was purchase from Addgene (Plasmid \#40173). Luminescence was measured using the Dual-Glo Luciferase Assay System (Promega) and the GloMax ${ }^{\circ}$ 20/ 20 Luminometer (Promega).

\section{Statistical analysis}

The experiments were performed at least three times for a reliable application of statistics. Statistical analysis was performed with GraphPad Prism software. Values are presented as mean \pm SD. ANOVA or Student's $\mathrm{T}$ test were used to evaluate the statistical significance.

\section{Results}

Extracellular acidosis promotes SOX2 expression in melanoma cells contributing to OxPhos metabolism

We have previously investigated the role of acidic tumor microenvironment in the acquisition of an aggressive [22] and chemo-resistant [23] phenotype in melanoma cells and found a closely related metabolic reprogramming that switches acidic cells to a more oxidative metabolism [7]. In this context, we hypothesized that the expression of the transcription factor SOX2 might be modulated by $\mathrm{pH}$ variations of tumor extracellular microenvironment and have a role in this metabolic adaptation. Flow cytometry and western blot analyses (Fig. 1a and b) showed increased SOX2 expression in A375-M6 melanoma cells exposed to acidic medium ( $\mathrm{pH}$ 6.7) compared to control ( $\mathrm{pH} 7.4$ ). We also observed that SOX2 expression was strictly regulated by $\mathrm{pH}$ variations: while $\mathrm{pH} 7.0$ was not sufficient to increase SOX2 expression, we obtained a maximal 
a

C
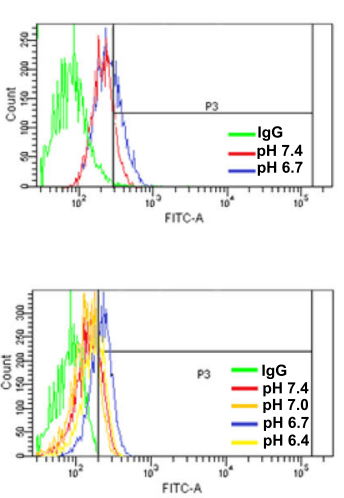

e

h
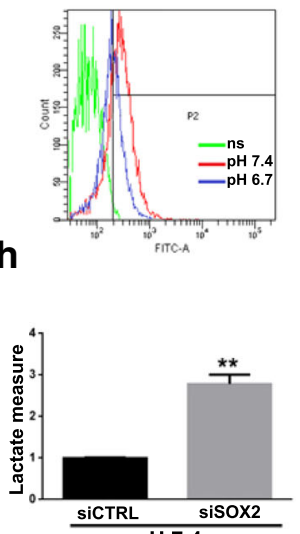

j

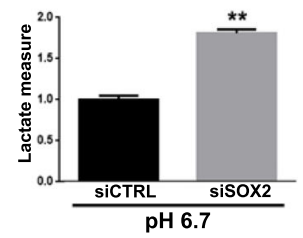

b
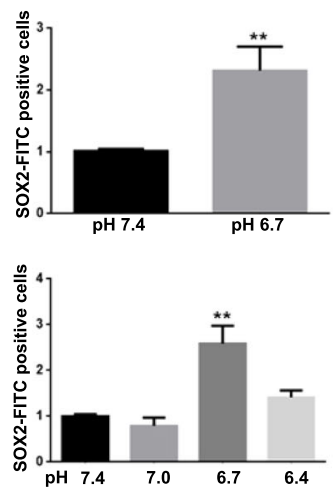

f

d

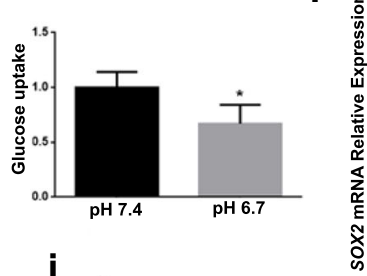

i

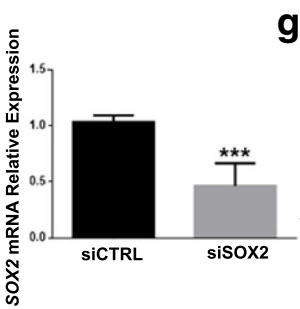

g
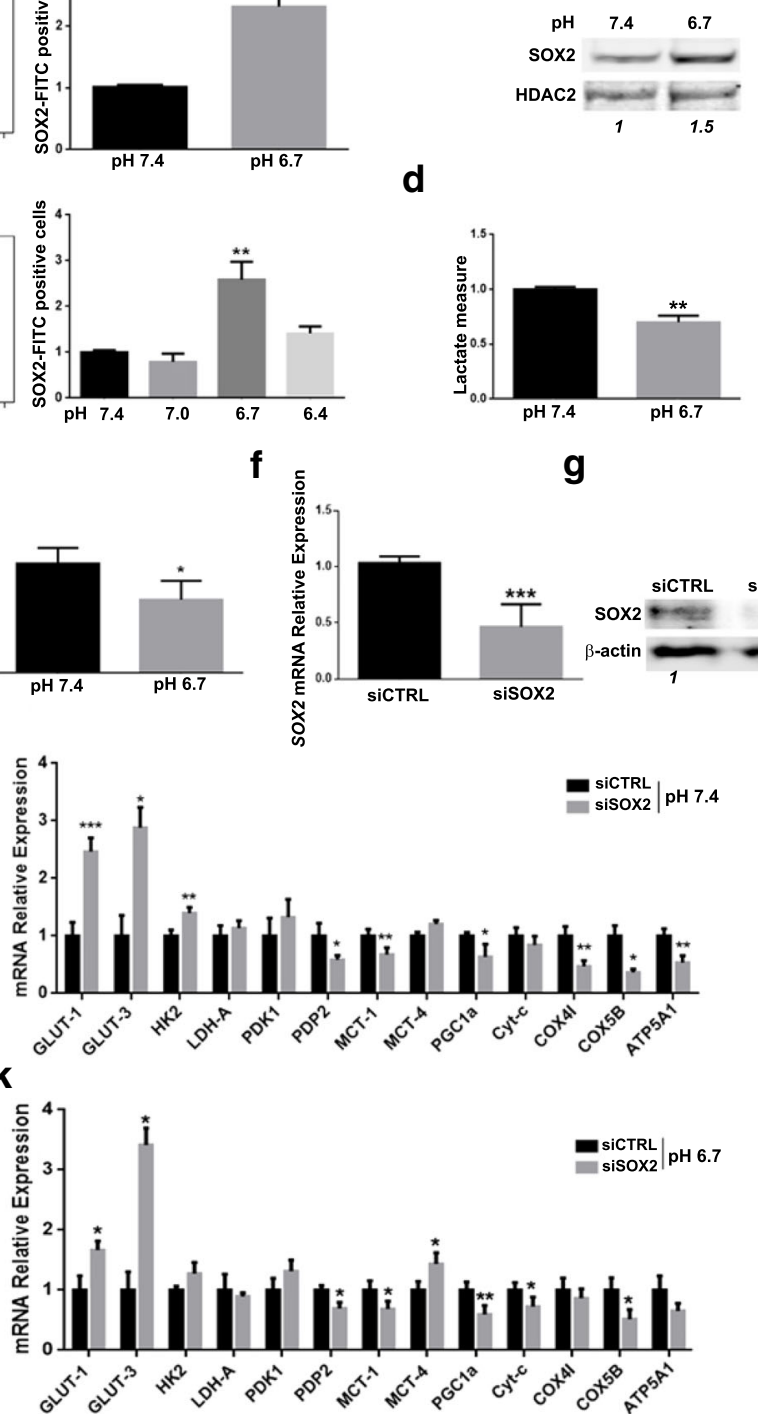

Fig. 1 SOX2 is up-regulated by extracellular acidosis and its inhibition increases the glycolytic metabolism in A375-M6 melanoma cells. a, b) Representative flow cytometry plot (left) and relative quantification chart (right) (a) and Western blot (b) of SOX2 in A375-M6 cells exposed for 24 h to standard pH 7.4 and acidic pH6.7. $p<0.05$, T-test. $N=3$. HDAC2 was used as loading control of nuclear protein fraction. Quantification of SOX2 protein expression in shown in italic. c) Representative flow cytometry plot (left) and relative quantification chart (right) of SOX2 level variation along with $\mathrm{pH}$ values. $p<0.01$, T-test, $\mathrm{N}=3$. d) Quantification of lactate production by A375-M6 cells exposed for $24 \mathrm{~h}$ to standard ( $\mathrm{pH}$ 7.4) or acidic ( $\mathrm{pH}$ 6.7) conditions. $p<0.01$, T-test, $N=3$. e) Representative flow cytometry plot (left) and quantification of glucose uptake (right) in A375-M6 cells exposed for $24 \mathrm{~h}$ to standard ( $\mathrm{pH}$ 7.4) or acidic ( $\mathrm{pH}$ 6.7) conditions. $p<0.05$, T-test, $\mathrm{N}=3$. $\mathbf{f}, \mathbf{g}$ ) Quantitative Real Time PCR ( $\mathrm{PPCR}$ ) (f) and Western blot (g) of SOX2 in A375-M6 silenced for SOX2 (siSOX2) compared to control (siCTRL). Quantification of SOX2 protein is shown in italic. $\beta$-actin used as loading control. $p<0.01$, T-test. $N=3$. h) Quantification chart of lactate production by A375-M6 siSOX2 compared to siCTRL in standard condition ( $\mathrm{pH}$ 7.4) $p<0.01$, T-test, $N=3$. i) qPCR of a panel of glycolysis- and OxPhos-related genes of A375-M6 in standard condition (pH 7.4) silenced for SOX2 (siSOX2) compared to control (siCTRL). ${ }^{*} p<0.05,{ }^{* *} p<0.01$; ${ }^{* * *} p<0.001$, T-test, $N=3$. j) Quantification chart of lactate production by acidosis-exposed (pH 6.7) siSOX2 A375-M6 compared siCTRL. $p<0.01$, T-test, $N=3$. k) qPCR analysis of a panel of glycolysis- and OxPhos-related genes of acidosis-exposed (pH 6.7) A375-M6 siSOX2 compared siCTRL. ${ }^{*} p<0.05,{ }^{* *} p<0.01$, ${ }^{* * *} p<0.001, \mathrm{~T}$-test. $N=3$

level at $\mathrm{pH}$ 6.7, but further medium acidification up to pH 6.4 restored SOX2 expression at control level (Fig. 1c). As previously reported [7], extracellular acidosis induces a metabolic shift towards OxPhos with a simultaneous slowdown of the glycolytic pathway, as confirmed by the reduced lactate production (Fig. 1d) and glucose uptake (Fig. 1e) by acidosis-exposed melanoma cells compared to control. To understand whether SOX2 up-regulation in acidic melanoma cells might be implicated in the reconversion to OxPhos 
metabolism, SOX2 was silenced (Fig. If and g). Lowering SOX2 level in melanoma cells, besides causing a decrease in cell proliferation (Additional file 1: Figure S1a) in accordance with the literature [13], correlated with a more glycolytic metabolism, as confirmed by augmented lactate production (Fig. 1h) and the increment of glycolytic gene expression together with a reduction of oxidative ones (Fig. 1i and Additional file 1: Figure S2). This change in metabolism elicited by SOX2 silencing was also evident in melanoma cells exposed to extracellular acidosis ( $\mathrm{pH}$ 6.7) (Fig. $1 \mathrm{j}$ and k), confirming the contribution of SOX2 to the OxPhos metabolic adaptation. Indeed, SOX2 silencing in melanoma cells, under both standard and acidic conditions, favored an enhanced expression of glucose transporters GLUT-1 and GLUT-3. Further, hexokinase isoform 2 (HK2), which catalyzes the rate-limiting first step of glycolysis, was stimulated upon SOX2 silencing in either standard or acidic cells, although in acidic cells does not reach a significantly level (Fig. 1i and Additional file 1: Figure S2). To evaluate the activity of pyruvate dehydrogenase $(P D H)$ enzyme, which drives the pyruvate enter into TCA cycle linking glycolysis to OxPhos, we tested the expression of two enzymes that regulate $P D H$ : the activating pyruvate dehydrogenase phosphatase 2 (PDP2) and the inhibiting pyruvate dehydrogenase kinase 1 (PDK1). In SOX2-silenced control and acidic melanoma cells, we observed reduction of $P D P 2$ and an appreciable increase of PDK1 (Fig. 1i and $\mathrm{k}$ ), suggesting an impaired mitochondrial OxPhos. Crucial for acidosis-adapted tumor cells, which have reprogrammed their metabolic phenotype to OxPhos, is the up-regulation of monocarboxylate transporter 1 (MCT1), a promoter of lactate influx. Instead, tumor cells prevalently relying on Warburg metabolism up-regulate monocarboxylate transporter 4 (MCT4) expression, a promoter of lactate efflux, in order to prevent intracellular acidification and subsequent cell death. We observed a MCT1 reduction and MCT4 increase in SOX2-depleted melanoma cells (Fig. 1i and k). Despite significant variations were obtained only under acidic condition, we believe that MCT1 reduction with an unchanged level of MCT4 observed in standard condition might be also indicative of a net lactate discharge. A dynamic test of lactate production (Fig. 1j) confirmed that SOX2 silencing in acidosis-exposed melanoma cells reverted OxPhos to a more glycolytic metabolism, further confirmed by the downregulation of OxPhos-related enzymes, peroxisome proliferator-activated receptor gamma coactivator $1-\alpha$ (PGC1 $)$, cytochrome-c (Cyt-c), cytochrome c oxidase subunit 4 isoform 1 (COX4I), cytochrome c oxidase subunit 5B (COX5B), ATP Synthase F1 Subunit Alpha

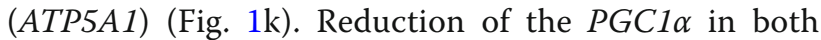

control and acidic-exposed melanoma cells is of special importance considering its activity in mitochondrial biogenesis and respiration in cancer cells tightly related to malignancy [24].

\section{Metabolic drugs sustain SOX2 contribution to OxPhos metabolism in acidic A375-M6 melanoma cells}

To further confirm the metabolic effects of SOX2 downregulation, we tested the efficacy of two metabolic drugs in control and SOX2-silenced A375-M6 melanoma cells maintained in standard condition $(\mathrm{pH}$ 7.4) or exposed to extracellular acidosis ( $\mathrm{pH}$ 6.7). We used 2-Deoxy-D-glucose (2-DG, $50 \mathrm{mM}$ ), a glucose analog which competitively inhibits glucose uptake and blocks the first critical step of glucose metabolism [25], and the mitochondrial respiration poison Metformin, a well-known antidiabetic drug that targets the complex I of the respiratory chain [26]. Treatment with 2-DG predisposed control A375-M6 grown in standard condition ( $\mathrm{pH} 7.4)$ to cell death ( 25\%), but these cytotoxic effects were almost doubled in siSOX2 melanoma cells $(\sim 45 \%$ cell death), suggesting their enhanced glycolytic dependency (Fig. 2a). On the contrary, Metformin treatment did not affect cell viability neither in control nor in siSOX2 cells $(\sim 2.5$ and $11 \%$ cell death, respectively), demonstrating insensitiveness to OxPhos inhibitors (Fig. 2a and b, pH 7.4). Upon melanoma cell exposure to extracellular acidosis, 2-DG exerted less cytotoxic effects in control cells $(\sim 18.5 \%)$ compared to what observed under standard $\mathrm{pH}$ condition, but induced a rapid enhancement of cell death in siSOX2 cells $(\sim 44.5 \%)$, disclosing a reverted attitude of these cells to glycolytic metabolism. On the other hand, Metformin was effective on acidic control cells ( $\sim 62.9 \%$ cell death), as expected, but its cytotoxic effects were significantly reduced in acidic siSOX2 cells ( $41.8 \%)$, highlighting a reduced OxPhos metabolism in these cells (Fig. 2a and b, $\mathrm{pH}$ 6.7). These results are consistent with our hypothesis that SOX2 down-regulation promotes a glycolytic metabolism, sensitizing melanoma cells to 2-DG and, at the same time, reducing the cytotoxic effect of Metformin.

\section{Modulation of SOX2 expression and metabolic adaptation of SSM2c and 501-Mel melanoma cells under standard conditions}

To confirm the correlation between SOX2 and OxPhos metabolism and evaluate the occurrence of any metabolic variations, we modulated SOX2 expression in other two human melanoma cell lines, chosen among a panel of cell lines based on their SOX2 expression level: patient-derived SSM2c cells, that express high levels of SOX2, and 501-Mel cells, that express very low SOX2 levels (Fig. 3a). When SOX2 was silenced in 


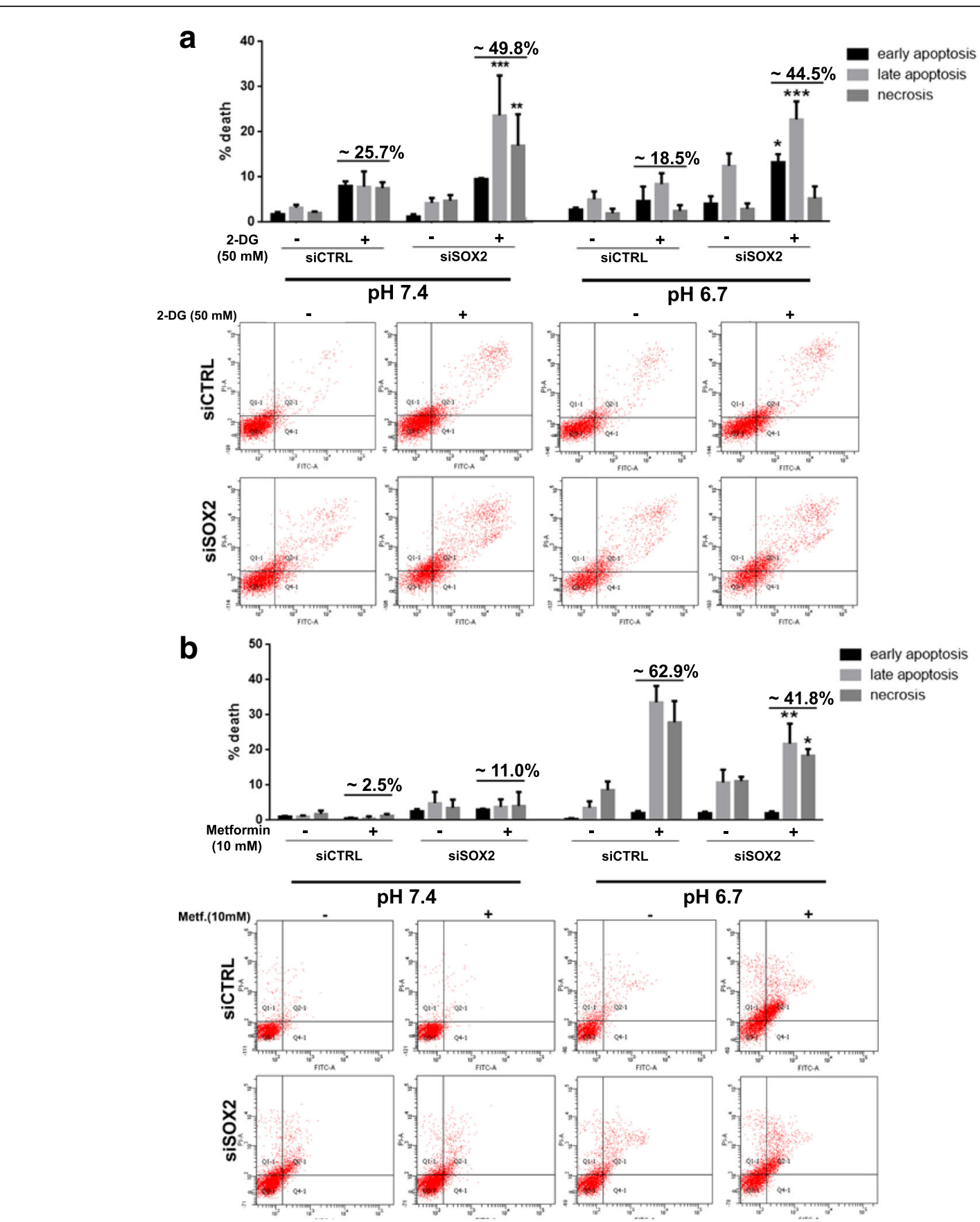

Fig. 2 SOX2 silencing in A375-M6 melanoma cells alters the efficacy of the metabolic drugs 2-DG and Metformin. a) Quantification chart (upper panel) and representative pictures (lower panel) of Annexin V/PI analysis of A375-M6 treated for $24 \mathrm{~h}$ with the anti-glycolytic drug 2-DG (50 mM) under standard ( $\mathrm{pH} \mathrm{7.4)} \mathrm{or} \mathrm{acidic} \mathrm{(pH} \mathrm{6.7)} \mathrm{conditions.} \mathrm{b)} \mathrm{Quantification} \mathrm{chart} \mathrm{(upper} \mathrm{panel)} \mathrm{and} \mathrm{representative} \mathrm{pictures} \mathrm{(lower} \mathrm{panel)} \mathrm{of} \mathrm{Annexin} \mathrm{V/}$ $\mathrm{Pl}$ analysis of A375-M6 treated for $24 \mathrm{~h}$ with the anti-OxPhos drug Metformin (10 mM) under standard $(\mathrm{pH} 7.4)$ or acidic ( $\mathrm{pH}$ 6.7) conditions. ${ }^{*} p<$ $0.05,{ }^{* *} p<0.01,{ }^{* * *} p<0.001$, Two-way ANOVA (statistical analysis compares for each phase- early apoptosis, late apoptosis, and necrosis- siSOX2 versus the respective untreated or treated siCTRL). $N=3$

high SOX2-expressing SSM2c cells (Fig. 3b and c), besides a decrease in cell proliferation (Additional file 1: Figure S1b), we observed a significant increase of lactate production (Fig. 3d) and of glycolytic genes, such as GLUT-1, HK2, lactate dehydrogenase A (LDH-A), PDK-
1, MCT-4, coupled with a PGC1 $\alpha$ reduction (Fig. 3e and Additional file 1: Figure S2). Instead, when low SOX2-expressing 501-Mel were forced to express SOX2 (Fig. 3f and g), we observed, along with increased cell proliferation (Additional file 1: Figure S1c), a decreased release of 


\section{a}
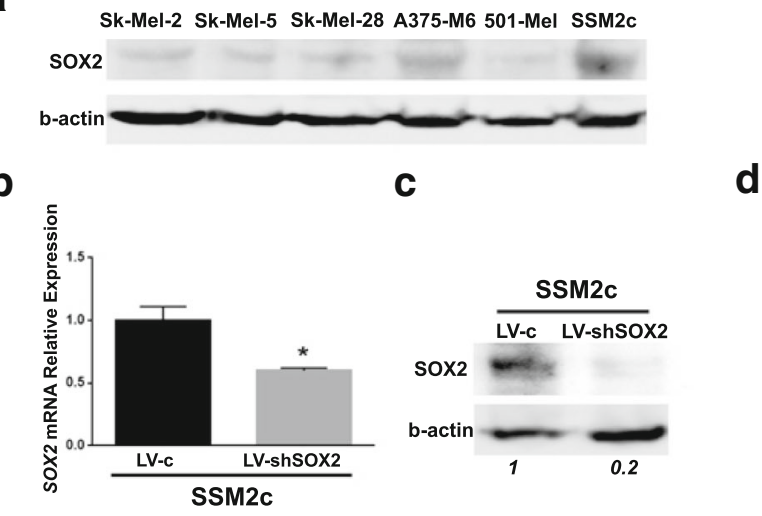

d

e

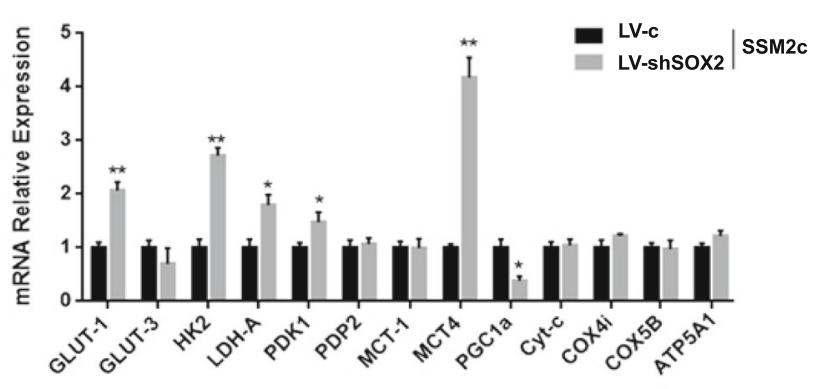

f

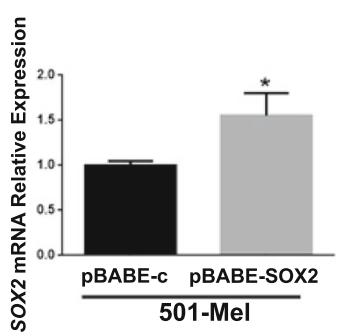

g

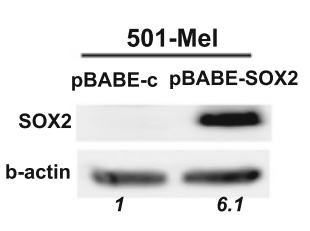

h

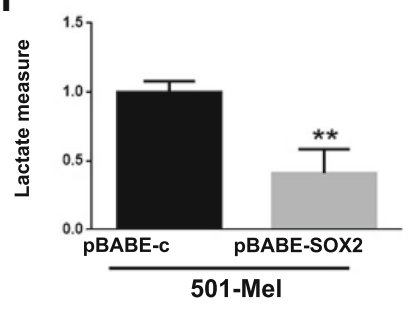

i

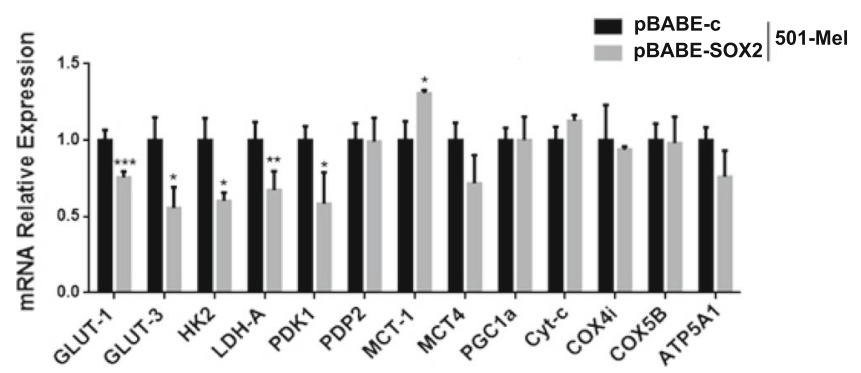

Fig. 3 Modulation of SOX2 expression induces metabolic changes in SSM2C and in 501-Mel melanoma cells. a) Western blot analysis of SOX2 expression in SK-MEL-2, SK-MEL-5, SK-MEL-28, A375-M6, 501-Mel and SSM2c melanoma cells. $\beta$-actin was used as loading control. b, c) qPCR (b) and Western blot (c) of SOX2 in SSM2C silenced for SOX2 (LV-shSOX2) compared to control (LV-c). Quantification of SOX2 protein is shown in italic. $p<0.05$, T-test. $N=3$. d) Quantification chart of lactate production of SSM2c LV-shSOX2 compared to LV-c. $p<0.05, T$-test, $N=3$. e) qPCR of a panel of glycolysis- and OxPhos-related genes in SSM2C LV-shSOX2 compared to LV-c. ${ }^{*} p<0.05,{ }^{* *} p<0.01,{ }^{* * *} p<0.001$, T-test. N $=3$. f, g) qPCR (f) and Western blot (g) of SOX2 in 501-Mel with SOX2 overexpression (pBABE-SOX2) compared to control (pBABE-c). Quantification of SOX2 protein is shown in italic. $p<0.01$, T-test. $N=3$. h) Quantification chart of lactate production of 501-Mel pBABE-SOX2 compared to pBABE-c. $p<0.01, \mathrm{~T}$-test, $N=3$. i) qPCR of a panel of glycolysis- and OxPhos-related genes in 501-Mel pBABE-SOX2 compared to pBABE-c. ${ }^{*} p<0.05,{ }^{* *} p<0.01,{ }^{* * *} p<0.001$, T-test. $N=3$

lactate in their media (Fig. 3h) and down-regulation of glycolytic genes, such as GLUT-1, GLUT-3, HK2, LDH-A, $P D K-1$, (Fig. $3 \mathrm{i}$ and Additional file 1: Figure S2).
Furthermore, ectopic SOX2 expression in 501-Mel increased the expression of $M C T-1$, which generally correlates with oxidative metabolism, but did not affect the 
other OxPhos-related genes (Fig. 3i and Additional file 1: Figure S2). All together, these findings confirm the role of SOX2 in OxPhos control.

\section{SOX2-driven metabolic adaptation to OxPhos is due to HIF1a pathway disruption}

Given the results obtained after modulation of SOX2 expression in melanoma cell lines grown in acidic and standard conditions, we hypothesized the involvement of HIF1 $\alpha$ transcription factor in SOX2-driven metabolic adaptation. Indeed, our findings indicate that low levels of SOX2 correlate with a blockage of the TCA cycle mediated by either a down-regulation of $P D P 2$ or an up-regulation of $P D K 1$ genes that, coupled with a higher lactate production and the modulation of GLUTs, HK2, LDH-A and MCTs genes, lead to the switch toward anaerobic glycolysis. In addition, we already know that melanoma cell exposure to extracellular acidosis deeply inhibits HIF1 $\alpha$ expression [21], leaving tumor cells to acquire an OxPhos phenotype, probably under the influence of the increased SOX2 expression, and able to maximize energy efficiency with the available resources. Adaptation to extracellular acidosis elicits a reduction of the energy sensor AMP-activated protein kinase (AMPK), which inhibits anabolic pathways and induces cell cycle slow down, dampening ATP consumption [7]. Acidosis-driven HIF1 $\alpha$ inhibition may suggest that, in certain circumstances, acidosis more than hypoxia could have a role in malignant progression. To confirm our hypothesis, we performed a bioinformatic analysis for the presence of putative SOX2-binding sites (BS) in the HIF1A promoter, and found two overlapping putative SOX2 BS in a region encompassing -929/-917bp upstream the transcription start site (TSS) (Fig. 4a). Chromatin Immunoprecipitation (ChIP) showed that SOX2 binds to the HIF1A promoter in that region (Fig. 4a), indicating that HIF1A is a direct downstream target of $S O X 2$. To determine the effect of SOX2 binding on HIF1A promoter, SOX2 was transfected with the HIF1A promoter $(-2159 \mathrm{bp} /+49 \mathrm{bp})$ containing the putative SOX2-BS driven by a luciferase reporter. Luciferase assay showed that SOX2 decreased the activity of the reporter in a dose-dependent manner (Fig. 4b), indicating that SOX2 inhibits HIF1 $\alpha$ expression by directly binding to its promoter. Thus, it was reasonable to evaluate if any HIF1 $\alpha$ variations occurred in response to SOX2 modulation in our cellular models. SOX2 silencing in SSM2c and A375-M6 increased the expression of HIF1 $\alpha$ mRNA and protein (Fig. 4c-f). Consistently, overexpression of SOX2 in 501-Mel melanoma cells drastically reduced HIF $1 \alpha$ mRNA and protein expression (Fig. $4 \mathrm{~g}$ and $\mathrm{h}$ ). These results indicate that SOX2 negatively regulates HIF $1 \alpha$ in melanoma cells. It should be considered that such variations have been observed under normoxic conditions, when HIF1 $\alpha$ is generally not completely stabilized. These HIF $1 \alpha$ variations might be enough for the metabolic changes observed in our experimental models of SOX2-manipulated melanoma cells.

\section{Discussion}

Cancer cells are characterized by a deregulated metabolism since, unlike normal cells, they largely depend on glycolysis even in the presence of oxygen, a phenomenon referred to as "Warburg effect" or aerobic glycolysis [3]. Nevertheless, cancer metabolism does not exclusively depend on aerobic glycolysis. Indeed, tumor cells can rather shift between different metabolic phenotypes or be in a hybrid state utilizing both glycolytic and oxidative metabolism [27]. This plasticity is also referred to cancer bioenergetics and contributes to positively select cancer cells in order to survive besides any environmental changes and hostile conditions [8]. We and others have already reported that the acidic microenvironment, that characterizes most of solid tumors and is associated with aggressive tumor phenotypes [22], favors OxPhos at the expense of glycolysis [7, 28-32]. Here we correlate for the first time SOX2 expression in acidic melanoma cells with a more oxidative metabolism, that is in turn associated with tumor progression and poor prognosis. In this regard, OxPhos metabolism has recently regained its role in cancer progression, given its association with occurrence of chemo-resistance and development of metastasis [27]. Moreover, despite the existence of controversial opinions [33], recent studies suggest that cancer stem cells are more reliant upon an oxidative metabolism than the non-stem bulk in different tumor types, including leukemia, ovarian, pancreatic, and breast cancer. OxPhos metabolism has been also shown to be privileged by circulating tumor cells compared to primary tumor cells of melanoma and breast cancers [24], and to be correlated with chemo-resistance in glioma [34], lung [35], pancreatic [36], prostate [37], and ovarian cancers [38]. Furthermore, several cases of metabolic shift to OxPhos following targeted therapies have been reported [39]. This is the case of melanomas carrying activating $B R A F$ mutations, where $B R A F$ inhibitors induce $P G C 1 \alpha$, a master regulator of mitochondrial biogenesis, which in turn promotes oxidative metabolism [40].

By exploiting an in vitro model of extracellular acidosis, we demonstrated that SOX2 is induced by an acidic microenvironment and, importantly, that SOX2 depletion in acidic melanoma cells reprograms their metabolism to a more glycolytic phenotype, also reducing OxPhos-related genes that characterize 


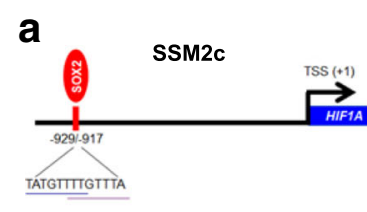

C

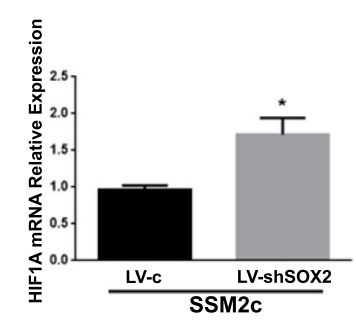

$\mathbf{e}$

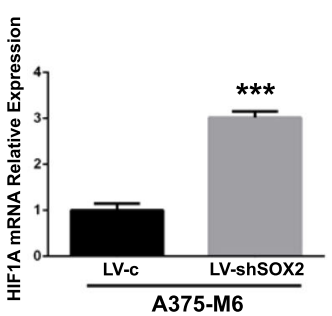

$\mathbf{g}$

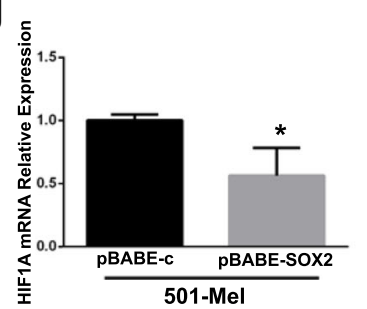

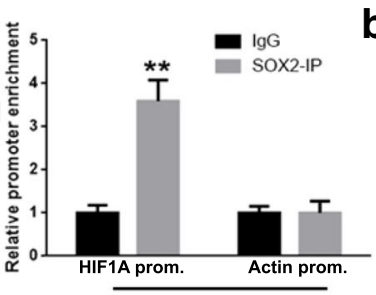

d

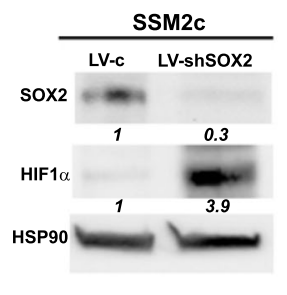

f

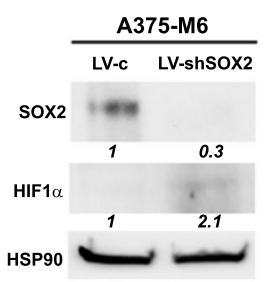

h

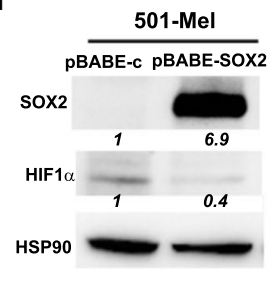

Fig. 4 SOX2 regulates HIF1a expression in SSM2C, A375-M6 and 501-Mel melanoma cells. a) (Left) Representative image of HIF1A promoter showing the sequence and the position of the putative SOX2-binding sites (BS) relative to the transcriptional start site (TSS). (Right) Chromatin Immunoprecipitation (ChIP) assay showing SOX2 binding at HIFIA promoter; Actin promoter was used as negative control and set to 1. $p<0.01$, Two-way ANOVA. b) Quantification of dual-luciferase reporter assay in SSM2c cells. Relative luciferase activities were Firefly/Renilla ratios, with the level induced by control equated to 1. Data represent mean \pm s.e.m. $p<0.001$ vs control, One-way ANOVA. $N=4$. c, d) qPCR (c) and Western blot (d) of HIF1a in SSM2C LV-shSOX2 compared to LV-c. Quantification of SOX2 and HIF1a protein is shown in italic. $p<0.01, T$-test. $N=4$. e, $\mathbf{f}) \mathrm{qPCR}$ (e) and Western blot (f) of HIF1a in A375-M6 LV-shSOX2 compared to LV-c. Quantification of SOX2 and HIF1a protein is shown in italic. $p<0.05$, T-test. $N=4 . \mathbf{g}, \mathbf{h}$ ) qPCR (g) and Western blot (h) of HIF1a in 501-Mel pBABE-SOX2 compared to pBABE-c. Quantification of SOX2 and HIF1a protein is shown in italic. $p<0.01$, T-test. $N=4$. HSP90 was used as loading control

acidosis-exposed melanoma cells. The reprogramming toward a more glycolytic profile is also evident in SOX2-silenced cells grown in standard condition. A tightly correlation between SOX2 and OxPhos emerges when SOX2-silenced melanoma cells, either grown in acidic or standard $\mathrm{pH}$ medium, are treated with 2-DG and Metformin. 2-DG targets glucose metabolism inducing a decrease of ATP generation, whereas Metformin blocks complex I of the respiratory chain. Interestingly, epidemiological and retrospective studies have revealed a lower incidence of cancer and better outcomes in diabetic patients taking Metformin compared to non-diabetics or diabetics using alternative drugs [41]. We found that 2-DG promotes cell death in SOX2-silenced cells grown in standard $\mathrm{pH}$ conditions and, most importantly, also in acidic melanoma cells depleted of SOX2. On the other hand, Metformin was effective only in acidosis-exposed cancer cells, since its efficacy is significantly reduced in SOX2-silenced cells, even though a further cell death reduction could be expected. This could be probably due to the high levels 
of SOX2 in acidic melanoma cells associated with an only partial SOX2 silencing efficacy.

To better understand SOX2 contribution in OxPhos metabolism, we determined metabolic markers in SSM2c cells, characterized by high SOX2 levels, and in 501-Mel, which show low/no SOX2 expression, upon knock-down or ectopic expression of SOX2, respectively. We confirmed the ability of SOX2 to contribute to an oxidative metabolism. Indeed, SOX2 knock-down leads to the suppression of the master regulator of mitochondrial metabolism $P G C 1 \alpha$, a phenomenon associated with the promotion of critical steps of the glycolytic pathway, i.e. GLUTs, HK2, PDP2/PDK1 axis and LDH-A. Furthermore, SOX2 silencing induces a switch of $M C T$ genes from type 1 to type 4 , indicating a preferred lactate efflux characteristic of a glycolytic metabolism. Consistently, in SOX2 overexpressing 501-Mel cells the reduction of most glycolytic markers came together with a promotion of $M C T-1$, a promoter of lactate influx. The clinical importance of MCT expression levels derived from their tightly correlation with shorter overall survival of advanced melanoma patients [42].

SOX2 has been already associated with tumor initiation, growth, drug resistance, and metastasis. Chemoresistant cancer cells that appear to preferably exploit oxidative metabolism, have been also associated with enhanced SOX2 expression in gastric, lung, prostate, colorectal [11], and breast [43] cancers. These findings prompted us to verify whether SOX2 influence in cell metabolism might be related to HIF1 $\alpha$ activity, considering that HIF $1 \alpha$ strongly induces a glycolytic phenotype. Our results indicate that HIF1 $\alpha$ and SOX2 are inversely correlated in normoxic condition, and this effect might be functionally sufficient to reprogram melanoma cells toward OxPhos. This is likely true mainly in conditions when SOX2 exceeds HIF $1 \alpha$ in terms of protein expression, as in the case of acidosis-exposed melanoma cells. Instead, under hypoxia, HIF1 $\alpha$ stabilization, despite the presence of SOX2 [44], likely represents the leading factor that causes cancer cell metabolic switch to anaerobic glycolysis. Among the so-called non-canonical HIF1 $\alpha$ regulation [45], it is quite interesting to recall that an increased lactate production is able to promote HIF1 $\alpha$ stabilization, although the mechanism has not been yet clarified [46]. Thus, the lactate increase observed in SOX2-silenced acidic and non-acidic melanoma cells could be able to contribute to HIF1 $\alpha$ expression and glycolytic re-conversion. Furthermore, quite recently it was demonstrated that HIF $1 \alpha$ represses PGC1 $\alpha$ expression in renal cell carcinoma, suggesting a regulatory loop among these transcriptional factors, involving oxygen sensing to mitochondrial biogenesis [47]. This is in line with our findings, i.e. PGC1 $\alpha$ reduction and $\mathrm{HIF} 1 \alpha$ promotion upon SOX2 silencing.

\section{Conclusions}

In conclusion, with this study we would propose a thigh correlation between SOX2 expression and OxPhos metabolism in melanoma cells, under a condition of reduced HIF $1 \alpha$ expression. Oxidative metabolism might be of a crucial importance for melanoma progression. Indeed, cancer cells may take advantage of this metabolic reprogramming toward OxPhos contributing to the development of an aggressive tumor phenotype endowed with an enhanced drug resistance and metastatic ability [48].

\section{Additional file}

Additional file 1: Figure S1. Growth curves of melanoma cells with SOX2 depletion or over-expression under standard and acidic condition. Figure S2. Western blotting of a panel of glycolysis- and OxPhos-related proteins after SOX2 silencing and over-expression in melanoma cells. (PDF $359 \mathrm{~kb}$ )

\section{Abbreviations}

2-DG: 2-Deoxiglucose; ATP5A1: ATP Synthase F1 Subunit Alpha; BS: binding site; COX4l: cytochrome c oxidase subunit 4 isoform 1; COX5B: cytochrome C oxidase subunit 5B; Cyt-c: cytochrome-C; GLUTs: glucose transporters; HIF1a: hypoxia-inducible factor a; HK2: hexokinase isoform 2; LDH-A: lactate dehydrogenase A; MCTs: monocarboxylate transporters; OxPhos: oxidative phosphorylation; PDK1: pyruvate dehydrogenase kinase 1; PDP2: pyruvate dehydrogenase phosphatase 2; PGC1a: peroxisome proliferator-activated receptor gamma coactivator 1-a; SOX2: sex-determining region Y (SRY)-Box2; TCA: tricarboxylic acid cycle; TSS: transcription starting site

\section{Acknowledgements}

not applicable.

\section{Funding}

this study was financially supported by Istituto Toscano Tumori (ITT) (Decreto Dirigenziale Regione Toscana n. 5254 of 04/12/2013 to LC), Ente Cassa di Risparmio di Firenze, Associazione Italiana per la Ricerca sul Cancro (AIRC) (grant IG-14184 to BS; fellowship to $\mathrm{EA}^{*}$, SP* and $\mathrm{AB}$ ) and Università degli Studi di Firenze.

Availability of data and materials

All data generated or analysed during this study are included in this published article and its supplementary information files.

\begin{abstract}
Authors' contributions
$L C, E A^{*}, S P^{*}$ and $B S$ designed the experiments and write the manuscript. EA* and $S P^{*}$ performed the experiments helped by SP, AB, JR and FB. All authors read and approved the final manuscript.
\end{abstract}

Ethics approval and consent to participate

Not applicable.

Consent for publication

Not applicable.

Competing interests

The authors declare that they have no competing interests.

\section{Publisher's Note}

Springer Nature remains neutral with regard to jurisdictional claims in published maps and institutional affiliations. 


\section{Author details}

"Department of Clinical and Experimental Biomedical Sciences "Mario Serio", Section of Experimental Pathology and Oncology, University of Florence, Viale G.B. Morgagni, 50, 50134 Florence, Italy. ${ }^{2}$ Core Research Laboratory, Institute for Cancer Research and Prevention (ISPRO), Florence, Italy. ${ }^{3}$ Center of Excellence for Research, Transfer and High Education DenoTHE University of Florence, Florence, Italy.

\section{Received: 19 July 2018 Accepted: 8 November 2018}

Published online: 22 November 2018

\section{References}

1. Hanahan D, Weinberg RA. Hallmarks of cancer: the next generation. Cell. 2011;144(5):646-74.

2. Vander Heiden MG, Cantley LC, Thompson CB. Understanding the Warburg effect: the metabolic requirements of cell proliferation. Science. 2009; 324(5930):1029-33.

3. Peppicelli S, Andreucci E, Ruzzolini J, Margheri F, Laurenzana A, Bianchini F, et al. Acidity of microenvironment as a further driver of tumor metabolic reprogramming. J Clin Cell Immunol. 2017:8:485.

4. Koukourakis MI, Giatromanolaki A, Harris AL, Sivridis E. Comparison of metabolic pathways between cancer cells and stromal cells in colorecta carcinomas: a metabolic survival role for tumor-associated stroma. Cancer Res. 2006:66(2):632-7.

5. Webb BA, Chimenti M, Jacobson MP, Barber DL. Dysregulated pH: a perfect storm for cancer progression. Nat Rev Cancer. 2011:11(9):671-7.

6. Peppicelli S, Bianchini F, Calorini L. Extracellular acidity, a "reappreciated" trait of tumor environment driving malignancy: perspectives in diagnosis and therapy. Cancer Metastasis Rev. 2014;33(2-3):823-32.

7. Peppicelli S, Toti A, Giannoni E, Bianchini F, Margheri F, Del Rosso M, et al. Metformin is also effective on lactic acidosis-exposed melanoma cells switched to oxidative phosphorylation. Cell Cycle. 2016;15(14):1908-18.

8. Zheng J. Energy metabolism of cancer: glycolysis versus oxidative phosphorylation (review). Oncol Lett. 2012;4(6):1151-7.

9. Kosmidis C, Baka S, Sapalidis K, Mixalopoulos N, Atmatzidis S, Koulouris H, et al. Melanoma from molecular pathways to clinical treatment: an up to date review. J Biomed. 2017;2:94-100.

10. Erdei $E$, Torres SM. A new understanding in the epidemiology of melanoma. Expert Rev Anticancer Ther. 2010;10(11):1811-23.

11. Wuebben EL, Rizzino A. The dark side of SOX2: cancer - a comprehensive overview. Oncotarget. 2017:8(27):44917-43.

12. Qureshi-Baig K, Ullmann P, Haan S, Letellier E. Tumor-initiating cells: a criTICal review of isolation approaches and new challenges in targeting strategies. Mol Cancer. 2017;16(1):40.

13. Santini R, Pietrobono S, Pandolfi S, Montagnani V, D'Amico M, Penachioni $J Y$, et al. SOX2 regulates self-renewal and tumorigenicity of human melanoma-initiating cells. Oncogene. 2014;33(38):4697-708.

14. Pietrobono S, Morandi A, Gagliardi S, Gerlini G, Borgognoni L, Chiarugi P, et al. Down-regulation of SOX2 underlies the inhibitory effects of the Triphenylmethane gentian violet on melanoma cell self-renewal and survival. J Invest Dermatol. 2016;136(10):2059-69.

15. Laga AC, Lai CY, Zhan Q, Huang SJ, Velazquez EF, Yang Q, et al. Expression of the embryonic stem cell transcription factor SOX2 in human skin: relevance to melanocyte and merkel cell biology. Am J Pathol. 2010;176(2):903-13.

16. Laga AC, Zhan Q, Weishaupt C, Ma J, Frank MH, Murphy GF. SOX2 and nestin expression in human melanoma: an immunohistochemical and experimental study. Exp Dermatol. 2011;20(4):339-45.

17. Chen PL, Chen WS, Li J, Lind AC, Lu D. Diagnostic utility of neural stem and progenitor cell markers nestin and SOX2 in distinguishing nodal melanocytic nevi from metastatic melanomas. Mod Pathol. 2013;26(1):44-53.

18. Girouard SD, Laga AC, Mihm MC, Scolyer RA, Thompson JF, Zhan Q, et al. SOX2 contributes to melanoma cell invasion. Lab Investig. 2012; 92(3):362-70.

19. Schaefer SM, Segalada C, Cheng PF, Bonalli M, Parfejevs V, Levesque MP, et al. Sox2 is dispensable for primary melanoma and metastasis formation. Oncogene. 2017;36(31):4516-24.

20. Cesarini V, Guida E, Todaro F, Di Agostino S, Tassinari V, Nicolis S, et al. Sox2 is not required for melanomagenesis, melanoma growth and melanoma metastasis in vivo. Oncogene. 2017;36(31):4508-15.
21. Andreucci E, Peppicelli S, Carta F, Brisotto G, Biscontin E, Ruzzolini J, et al. Carbonic anhydrase IX inhibition affects viability of cancer cells adapted to extracellular acidosis. J Mol Med (Berl). 2017;95(12):1341-53.

22. Peppicelli S, Bianchini F, Torre E, Calorini L. Contribution of acidic melanoma cells undergoing epithelial-to-mesenchymal transition to aggressiveness of non-acidic melanoma cells. Clin Exp Metastasis. 2014;31(4):423-33.

23. Ruzzolini J, Peppicelli S, Andreucci E, Bianchini F, Margheri F, Laurenzana A, et al. Everolimus selectively targets vemurafenib resistant BRAF(V600E) melanoma cells adapted to low pH. Cancer Lett. 2017:408:43-54.

24. LeBleu VS, O'Connell JT, Gonzalez Herrera KN, Wikman H, Pantel K, Haigis MC, et al. PGC-1a mediates mitochondrial biogenesis and oxidative phosphorylation in cancer cells to promote metastasis. Nat Cell Biol. 2014; 16(10):992-1003 1-15.

25. Pelicano $H$, Martin DS, Xu RH, Huang P. Glycolysis inhibition for anticancer treatment. Oncogene. 2006:25(34):4633-46.

26. Wheaton WW, Weinberg SE, Hamanaka RB, Soberanes S, Sullivan LB, Anso E, et al. Metformin inhibits mitochondrial complex I of cancer cells to reduce tumorigenesis. elife. 2014;3:e02242.

27. Yu L, Lu M, Jia D, Ma J, Ben-Jacob E, Levine H, et al. Modeling the genetic regulation of Cancer metabolism: interplay between glycolysis and oxidative phosphorylation. Cancer Res. 2017;77(7):1564-74.

28. Peppicelli S, Bianchini F, Calorini L. Dynamic scenario of metabolic pathway adaptation in tumors and therapeutic approach. Oncoscience. 2015;2(3):225-32.

29. Wu H, Ying M, Hu X. Lactic acidosis switches cancer cells from aerobic glycolysis back to dominant oxidative phosphorylation. Oncotarget. 2016; 7(26):40621-9.

30. Khacho M, Tarabay M, Patten D, Khacho P, MacLaurin JG, Guadagno J, et al Acidosis overrides oxygen deprivation to maintain mitochondrial function and cell survival. Nat Commun. 2014:5:3550

31. Mazzio EA, Boukli N, Rivera N, Soliman KF. Pericellular pH homeostasis is a primary function of the Warburg effect: inversion of metabolic systems to control lactate steady state in tumor cells. Cancer Sci. 2012;103(3):422-32.

32. Lamonte G, Tang X, Chen JL, Wu J, Ding CK, Keenan MM, et al. Acidosis induces reprogramming of cellular metabolism to mitigate oxidative stress. Cancer Metab. 2013:1(1):23.

33. Peiris-Pagès M, Martinez-Outschoorn UE, Pestell RG, Sotgia F, Lisanti MP. Cancer stem cell metabolism. Breast Cancer Res. 2016;18(1):55.

34. Oliva CR, Moellering DR, Gillespie GY, Griguer CE. Acquisition of chemoresistance in gliomas is associated with increased mitochondrial coupling and decreased ROS production. PLoS One. 2011;6(9):e24665.

35. Sun Y, Daemen A, Hatzivassiliou G, Arnott D, Wilson C, Zhuang G, et al. Metabolic and transcriptional profiling reveals pyruvate dehydrogenase kinase 4 as a mediator of epithelial-mesenchymal transition and drug resistance in tumor cells. Cancer Metab. 2014:2(1):20.

36. Viale A, Pettazzoni P, Lyssiotis CA, Ying H, Sánchez N, Marchesini M, et al. Oncogene ablation-resistant pancreatic cancer cells depend on mitochondrial function. Nature. 2014;514(7524):628-32

37. Ippolito L, Marini A, Cavallini L, Morandi A, Pietrovito L, Pintus G, et al. Metabolic shift toward oxidative phosphorylation in docetaxel resistant prostate cancer cells. Oncotarget. 2016;7(38):61890-904.

38. Dar S, Chhina J, Mert I, Chitale D, Buekers T, Kaur H, et al. Bioenergetic adaptations in Chemoresistant ovarian Cancer cells. Sci Rep. 2017:7(1):8760.

39. Ashton TM, McKenna WG, Kunz-Schughart LA, Higgins GS. Oxidative Phosphorylation as an Emerging Target in Cancer Therapy. Clin Cancer Res. 2018;24(11):2482-90.

40. Haq R, Shoag J, Andreu-Perez P, Yokoyama S, Edelman H, Rowe GC, et al. Oncogenic BRAF regulates oxidative metabolism via PGC1a and MITF. Cancer Cell. 2013;23(3):302-15.

41. Pernicova I, Korbonits M. Metformin--mode of action and clinical implications for diabetes and cancer. Nat Rev Endocrinol. 2014;10(3):143-56

42. Pinheiro $C$, Miranda-Gonçalves $V$, Longatto-Filho $A$, Vicente $A L$ Berardinelli GN, Scapulatempo-Neto C, et al. The metabolic microenvironment of melanomas: prognostic value of MCT1 and MCT4. Cell Cycle. 2016;15(11):1462-70

43. Mukherjee P, Gupta A, Chattopadhyay D, Chatterii U. Modulation of SOX2 expression delineates an end-point for paclitaxel-effectiveness in breast cancer stem cells. Sci Rep. 2017:7(1):9170.

44. Bae KM, Dai Y, Vieweg J, Siemann DW. Hypoxia regulates SOX2 expression to promote prostate cancer cell invasion and sphere formation. Am J Cancer Res. 2016;6(5):1078-88. 
45. LaGory EL, Giaccia AJ. The ever-expanding role of HIF in tumour and stromal biology. Nat Cell Biol. 2016;18(4):356-65.

46. Lu H, Dalgard CL, Mohyeldin A, McFate T, Tait AS, Verma A. Reversible inactivation of HIF-1 prolyl hydroxylases allows cell metabolism to control basal HIF-1. J Biol Chem. 2005;280(51):41928-39.

47. LaGory EL, Wu C, Taniguchi CM, Ding CC, Chi JT, von Eyben R, et al. Suppression of PGC-1a is critical for reprogramming oxidative metabolism in renal cell carcinoma. Cell Rep. 2015;12(1):116-27.

48. Ho J, de Moura MB, Lin Y, Vincent G, Thorne S, Duncan LM, et al. Importance of glycolysis and oxidative phosphorylation in advanced melanoma. Mol Cancer. 2012;11:76.

Ready to submit your research? Choose BMC and benefit from:

- fast, convenient online submission

- thorough peer review by experienced researchers in your field

- rapid publication on acceptance

- support for research data, including large and complex data types

- gold Open Access which fosters wider collaboration and increased citations

- maximum visibility for your research: over $100 \mathrm{M}$ website views per year

At $\mathrm{BMC}$, research is always in progress.

Learn more biomedcentral.com/submissions 